\title{
ASSISTÊNCIA DE ENFERMAGEM AO PACIENTE COM ACIDENTE VASCULAR ENCEFÁLICO
}

\section{NURSING ASSISTANCE TO PATIENTS WITH BRAIN VASCULAR ACCIDENT}

\author{
Maria Caroline Andrade Ribeiro ${ }^{1} *$ Maria Adriely Cunha Lima ${ }^{2}$ Ana Carolina Amorim \\ Oliveira $^{3} *$ Mércia Rocha Souza $^{4} *$ Raquel Santos Alves $^{5} *$ Fernanda Vasconcelos Prado $^{*}$ \\ Correia $^{6} *$ Larissa Ferreira Sales $^{7} *$ Victória Santos Alves $^{8} *$ Tatiane de Oliveira Santos $^{9} *$ \\ Halley Ferraro Oliveira ${ }^{10}$
}

\begin{abstract}
RESUMO
Objetivo: Identificar as principais condutas adotadas pela equipe de enfermagem diante de um paciente com Acidente Vascular Encefálico (AVE). Metodologia: Trata-se de uma revisão integrativa de artigos procurados através de descritores das bases de dados BVS, SciELO e PubMED, encontrando-se 12 estudos para atingir o objetivo proposto. Resultados: Observou-se que a equipe de enfermagem atuava por meio do reconhecimento de sinais e sintomas do AVE, realizando alguns testes, além da procura de fatores de risco, como fibrilação atrial, na história clínica do paciente. Essa atuação está relacionada ao fato do profissional de enfermagem, majoritariamente, ser responsável pela triagem dos pacientes. Nesse contexto, é válido mencionar a importante da equipe multidisciplinar para realizar a terapêutica do paciente com AVE rapidamente, minimizando assim as complicações desse evento. Conclusão: Nota-se a importância da assistência de enfermagem na identificação precoce do AVE, logo é necessário que esses profissionais tenham conhecimento desses sinais/sintomas, reduzindo assim a morbimortalidade desses pacientes. Palavras-chave: Acidente Vascular Encefálico; Assistência de Enfermagem; Enfermagem Centrada no Paciente.
\end{abstract}

\begin{abstract}
Objective: To identify as the main behaviors adopted by the nursing team in the face of a patient with a stroke. Methodology: This is an integrative review of articles sought through descriptors from the VHL, SciELO and PubMED databases, with 12 studies to achieve the proposed objective. Results: It was observed that the nursing team acted by recognizing the signs and symptoms of the stroke, performing some tests, in addition to looking for risk factors, such as atrial fibrillation, in the patient's clinical history. This performance is related to the fact that the nursing professional is mostly responsible for screening patients. In this context, it is worth mentioning an important member of the multidisciplinary team to carry out the therapy of the stroke patient quickly, thus minimizing complications of this event. Conclusion: Note the importance of nursing care in the early identification of stroke, so it is necessary that these professionals have knowledge of signs / signs, thus the morbidity and mortality of these patients. Key words: Brain stroke; Nursing Assistance; Patient-Centered Nursing.

\footnotetext{
1 Graduanda de enfermagem na Universidade Tiradentes (UNIT-SE). Orcid: http://orcid.org/0000-0003-2955-8844. E-mail: maria.caribeiro@souunit.com.br

${ }^{2}$ Graduanda de medicina na Universidade Tiradentes (UNIT-SE). Orcid: E-mail: mariaadrielycunha@hotmail.com

3 Graduanda de medicina na Universidade Tiradentes (UNIT-SE). Orcid: http://orcid.org/0000-0001-8132-1516. E-mail: ana.camorim@souunit.com.br

${ }^{4}$ Graduanda de enfermagem no Centro Universitário Maurício de Nassau (UNINASSAU-SE). Orcid: http://orcid.org/0000-00030380-324X. E-mail: mercia_rh@hotmail.com

5 Graduanda de enfermagem no Centro Universitário Maurício de Nassau (UNINASSAU-SE). Orcid: http://orcid.org/0000-00022673-7655. E-mail: raquel.santos.alves.uninassau@outlook.com

6 Graduanda de medicina na Universidade Tiradentes (UNIT-SE). Orcid: http://orcid.org/0000-0002-9110-3384. E-mail: fe.concelos@gmail.com

7 Graduanda de enfermagem na Universidade Tiradentes (UNIT-SE). Orcid: http://orcid.org/0000-0003-4480-9082. E-mail: larissa.fsales@souunit.com.br

8 Graduanda de enfermagem na Universidade Tiradentes (UNIT-SE). Orcid: http://orcid.org/0000-0001-6122-7952. E-mail: victorialvesantos@outlook.com

9 Graduada em enfermagem pela Universidade Tiradentes (UNIT-SE). Orcid: http://orcid.org/0000-0003-3836-2560. E-mail: enfatatisantos@gmail.com

10 Professor adjunto da Universidade Tiradentes (UNIT-SE). Orcid: http://orcid.org/0000-0003-0123-7395. E-mail: halleyoliveira62@gmail.com
} 


\section{INTRODUÇÃO}

O Acidente Vascular Encefálico (AVE) é representado por um infarto agudo do tecido nervoso central de um indivíduo, ou seja, após ruptura de vasos, isquemia cerebral devido a hipoperfusão, ou evento tromboembólico. Tal processo pode ocorrer devido a causas externas ao ambiente hospitalar ou até mesmo durante a hospitalização, sendo consequência de outra patologia ou procedimento realizado (1). Desse modo, o AVE configura-se como uma condição capaz de gerar consequências prejudiciais à saúde do indivíduo, podendo incapacitar ou até mesmo provocar a morte do paciente acometido, sobretudo no ambiente hospitalar $^{(2)}$.

Mediante esse contexto, as causas que podem desencadear o AVE são diversas, dentre elas encontram-se: presença de dislipidemias, possuir idade avançada, ser tabagista ou etilista, ter hábitos de vida sedentários ou ter problemas cardiovasculares (3) De acordo com estudos, fatores como problemas cardiovasculares são indicadores para o surgimento de AVE, por exemplo, têmse notado que elevadas taxas de hipertensão são bastante constantes em indivíduos na fase adulta e idosa. Sendo assim, percebe-se que o impacto negativo da doença se torna reduzido quando o tratamento é realizado de forma precoce ou imediata ${ }^{(4)}$.

De acordo com a American Heart Association/ American Stroke Association
(AHA/ASA, 2016), tendo como auxílio várias associações e entidades de saúde, identificouse uma diminuição no número de acidentes vasculares encefálicos em todo o mundo, no entanto, contrariando as expectativas dos profissionais de saúde, observou-se que o número da população que teve a enfermidade e obteve sequelas permanentes evoluiu muito (5).

Nesse sentido, identifica-se a crucial importância de boa assistência da equipe de enfermagem ao paciente diagnosticado com AVE desde o atendimento pré-hospitalar até o intra-hospitalar, a fim de potencializar melhores condições do quadro clínico do indivíduo. Cuidados que envolvem a avaliação fisiológica, administração de medicamentos, apoio psicológico e emocional, bem como reabilitação funcional podem estar envolvidos diretamente com a assistência de enfermagem ${ }^{(6,7)}$. Dessa forma, ocorre a redução de alterações sensóriomotora provenientes da condição, bem como estabelecimento de melhores condições de vida dos pacientes ${ }^{(7)}$.

O AVE é uma doença silenciosa, que afeta grande parte da população mundial devido a sua alta taxa de morbimortalidade dentre o grupo de doenças cardiovasculares existentes. Ao analisar essa temática, percebeu-se que existe a necessidade de abordar sobre a assistência de enfermagem ao paciente com AVE, a fim de enfatizar benefícios gerados 
após um cuidado de qualidade. Desta forma, surgiu a indagação de quais seriam as medidas adotadas pela equipe de enfermagem a fim de garantir uma assistência ao paciente de forma eficaz?

Sendo assim, o presente artigo tem como objetivo geral identificar quais são as condutas adotadas pela equipe de enfermagem diante de paciente com AVE; e os objetivos específicos são destacar os principais sinais e sintomas do AVE; analisar o impacto gerado mediante a execução da assistência de qualidade. Considera-se, que o estudo tenha grande relevância para os profissionais que atuam na área de enfermagem de urgência e emergência e acadêmicos da área da saúde. Uma vez que, há necessidade de discutir a temática devido tratar-se de um assunto de extrema importância para a sociedade.

\section{MÉTODOS}

Trata-se de uma revisão integrativa, com uma abordagem qualitativa, estruturada mediante as seguintes etapas: reflexão e construção da pergunta norteadora; seleção e busca de artigos; significação das informações a serem retiradas dos estudos selecionados; avaliação crítica dos trabalhos selecionados; discussão dos resultados e apresentação da revisão integrativa. Para a primeira etapa, as seguintes perguntas norteadoras foram elaboradas: "Quais as práticas utilizadas para a melhor assistência de enfermagem a pacientes com AVE? O enfermeiro possui habilidades necessárias para a assistência desses pacientes? ".

Foi realizada a busca dos artigos, baseada na análise dos dados disponibilizados na Biblioteca Virtual em Saúde (BVS), Scientific Electronic Library Online (SciELO), Público/editora MEDLINE (PubMED) foram utilizadas. Para isso, definiu-se os seguintes Descritores em Ciências da Saúde (DECS) “Acidente Vascular Encefálico" (D1), "Assistência de Enfermagem" (D2) e “Enfermagem Centrada no Paciente" (D3), os quais foram combinados com o auxílio do operador booleano AND. Primeiramente utilizou-se "D1" AND "D3”, em seguida, "D2" AND "D1" AND "D3", quando combinados forneceram um quantitativo de 32 artigos.

Foi definido como critérios de inclusão textos completos disponíveis online em português e/ou inglês, publicados entre 2015 a 2020. Já os de exclusão foram artigos que não apresentavam significativa relação com a temática abordada e estudos de domínio privado. Após isso, um total de 20 artigos foram selecionados nas bases.

Ao aplicar os critérios de elegibilidade, apenas 12 artigos foram selecionados, pois abordaram de maneira satisfatória as atribuições da enfermagem na assistência a pacientes com AVE. Por conseguinte, esses 
artigos foram submetidos à leitura prévia dos títulos e dos resumos, para melhor compreensão e identificação dos objetivos dos estudos e dos métodos utilizados. No segundo momento de leitura, por sua vez, foi realizada uma análise mais aprofundada, buscando identificar aspectos ligados intimamente com a temática abordada, após isso restaram apenas 10 estudos para compor a base de dados dessa revisão (Figura 1).

Por se tratar de uma pesquisa bibliográfica, os dados obtidos seguiram princípios éticos, segundo a Lei dos Direitos Autorais número 12.853/2013. As informações contidas neste estudo foram citadas de maneira fidedigna, conforme bibliografia selecionada e preconizada pelas normas Vancouver.

Figura 1 - Diagrama de fluxo PRISMA do estudo.
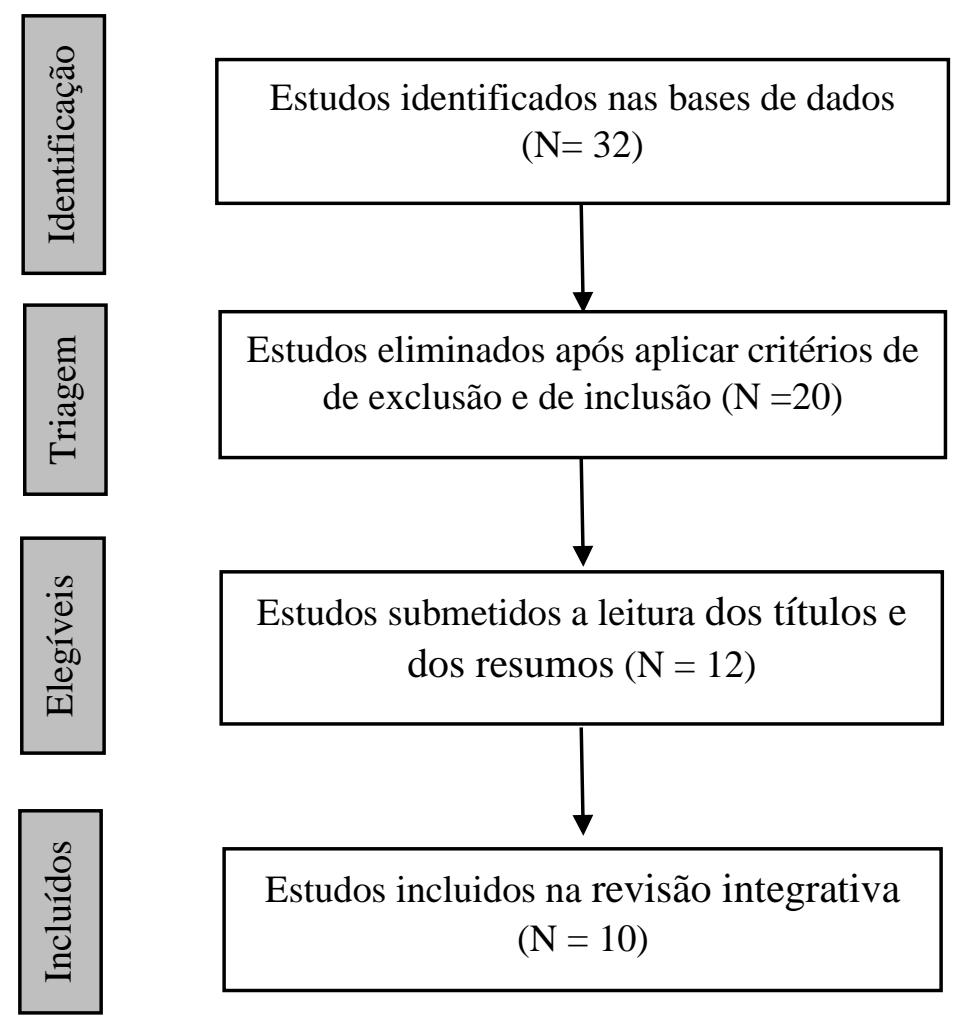

Fonte: dados da pesquisa, 2021.

\section{RESULTADOS}

A partir da busca de dados foram selecionados 10 artigos que atendiam aos critérios de inclusão, com destacado no Quadro 1.

Quadro 1 - Artigos selecionados em relação à questão norteadora da pesquisa.

\begin{tabular}{|l|l|l|l|}
\hline AUTORIA & TÍTULO & MÉTODO & \multicolumn{1}{c|}{ RESULTADOS } \\
\hline & & & Os fatores de risco, simulações e \\
\hline
\end{tabular}

https://doi.org/10.31011/reaid-2021-v.95-n.34-art.1001 Rev Enferm Atual In Derme v. 95, n. 34, 2021 e-021091 


\begin{tabular}{|c|c|c|c|}
\hline $\begin{array}{l}\text { Cumbler } \\
\text { MDE, } 2015\end{array}$ & $\begin{array}{l}\text { In-Hospital } \\
\text { Stroke }\end{array}$ & $\begin{array}{l}\text { Estudo de } \\
\text { caso }\end{array}$ & $\begin{array}{l}\text { etiologia de isquemia por acidente } \\
\text { vascular cerebral intra-hospitalar } \\
\text { são diferentes daqueles em } \\
\text { Protocolo de alerta de AVC no } \\
\text { hospital }\end{array}$ \\
\hline $\begin{array}{l}\text { Graça CSG, } \\
2019^{(3)}\end{array}$ & $\begin{array}{l}\text { Qualidade na abordagem e } \\
\text { tratamento do doente com } \\
\text { Acidente Vascular } \\
\text { Cerebral (AVC) Isquêmico }\end{array}$ & $\begin{array}{l}\text { Estudo } \\
\text { descritivo e } \\
\text { retrospectivo }\end{array}$ & $\begin{array}{l}\text { Na amostra de } 442 \text { doentes com } \\
\text { AVC isquêmico, } 55 \% \text { eram do sexo } \\
\text { masculino, a idade média foi de } \\
73.3 \text { anos e a prevalência de } \\
\text { comorbidades cardiovasculares foi } \\
\text { de } 90 \%\end{array}$ \\
\hline $\begin{array}{l}\text { Grilo JNF, } \\
2018^{(5)}\end{array}$ & $\begin{array}{l}\text { Melhoria da eficácia das } \\
\text { intervenções do enfermeiro } \\
\text { especialista } \\
\text { enfermagem }\end{array}$ & $\begin{array}{l}\text { Estudo } \\
\text { caso }\end{array}$ & $\begin{array}{l}\text { As intervenções da enfermagem de } \\
\text { reabilitação melhoram a promoção } \\
\text { do autocuidado, proporcionando } \\
\text { ganhos funcionais aos acometidos } \\
\text { por AVC }\end{array}$ \\
\hline $\begin{array}{ll}\text { Silva } & \text { DN, } \\
2020^{(7)} & \end{array}$ & $\begin{array}{l}\text { Cuidados de enfermagem a } \\
\text { vítima de AVC }\end{array}$ & $\begin{array}{l}\text { Revisão } \\
\text { sistemática }\end{array}$ & $\begin{array}{l}\text { Por meio deste estudo ficou } \\
\text { evidente a importância dos } \\
\text { cuidados prestados pelo enfermeiro } \\
\text { e sua equipe na assistência ao } \\
\text { paciente vítima de AVC. Podem } \\
\text { realizar intervenções na prevenção, } \\
\text { tratamento e reabilitação }\end{array}$ \\
\hline $\begin{array}{l}\text { Meneses PS, } \\
\text { et al., } 2019 \\
(8)\end{array}$ & $\begin{array}{l}\text { Abordagem } \\
\text { fisioterapêutica } \\
\text { tratamento de AVC }\end{array}$ & $\begin{array}{l}\text { Relato } \\
\text { caso }\end{array}$ & $\begin{array}{l}\text { Paciente, com diagnóstico de AVC } \\
\text { isquêmico, atendido na clínica } \\
\text { escola de fisioterapia da } \\
\text { Unicatólica. Colaborativo e } \\
\text { participante do relato de caso. } \\
\text { Aceitou bem todo o protocolo de } \\
\text { atendimento programado }\end{array}$ \\
\hline $\begin{array}{l}\text { Lima } \\
\text { ACMACC et } \\
\text { al, } 2016^{(9)}\end{array}$ & $\begin{array}{lr}\text { Diagnósticos } & \text { de } \\
\text { enfermagem em } & \text { pacientes } \\
\text { com acidente } & \text { vascular } \\
\text { cerebral: } & \text { revisão } \\
\text { integrativa } & \end{array}$ & $\begin{array}{l}\text { Estudo } \\
\text { descritivo }\end{array}$ & $\begin{array}{l}\text { O enfermeiro } \text { identifica } \\
\text { determinados sinais/sintomas } \\
\text { presentes nos pacientes com AVC, } \\
\text { além de realizar o cuidado após o } \\
\text { AVC, interferindo nos problemas } \\
\text { funcionais e psicossociais }\end{array}$ \\
\hline $\begin{array}{l}\text { Saltman AP, } \\
\text { et al., } 2015 \\
(10)\end{array}$ & $\begin{array}{l}\text { Cuidados e resultados de } \\
\text { pacientes com AVC no } \\
\text { hospital }\end{array}$ & $\begin{array}{l}\text { Estudo de } \\
\text { caso clínico }\end{array}$ & $\begin{array}{l}\text { Pacientes com AVC hospitalar em } \\
\text { comparação com aqueles com } \\
\text { derrame de início da comunidade } \\
\text { teve tempos de espera } \\
\text { significativamente mais longos do } \\
\text { reconhecimento dos sintomas para } \\
\text { neuroimagem apesar dos cuidados }\end{array}$ \\
\hline $\begin{array}{l}\text { Cumbler E, } \\
\text { et al., } 2014 .\end{array}$ & $\begin{array}{l}\text { Qualidade do atendimento } \\
\mathrm{e} \text { resultados para AVC }\end{array}$ & $\begin{array}{l}\text { Estudo } \\
\text { coorte }\end{array}$ & $\begin{array}{l}\text { Pacientes com AVC intra-hospitalar } \\
\text { apresentaram mais fatores de risco }\end{array}$ \\
\hline
\end{tabular}




\begin{tabular}{|c|c|c|c|}
\hline (11) & $\begin{array}{l}\text { isquêmico intra-hospitalar: } \\
\text { achados do National Get } \\
\text { With The Guidelines- } \\
\text { Stroke }\end{array}$ & retrospectivo & $\begin{array}{l}\text { tromboembólicos e experimentaram } \\
\text { AVC mais graves apesar de estarem } \\
\text { em cuidados da equipe } \\
\text { multidisciplinar }\end{array}$ \\
\hline $\begin{array}{l}\text { Lima RJ, et } \\
\text { al., 2020. }{ }^{(12)}\end{array}$ & $\begin{array}{l}\text { Resiliência, capacidade } \\
\text { funcional e apoio social de } \\
\text { pessoas com sequelas de } \\
\text { AVE }\end{array}$ & $\begin{array}{l}\text { Estudo } \\
\text { transversal }\end{array}$ & $\begin{array}{l}\text { Os participantes apresentaram mais } \\
\text { frequentemente o nível de } \\
\text { resiliência moderada }(64,8 \%) \text {, } \\
\text { dependência funcional }(93,6 \%) \text { para } \\
\text { realização das atividades diárias e } \\
\text { médio apoio social }(48,2 \%)\end{array}$ \\
\hline $\begin{array}{l}\text { Syafrita Y, et } \\
\text { al., } 2020 \text { (13) }\end{array}$ & 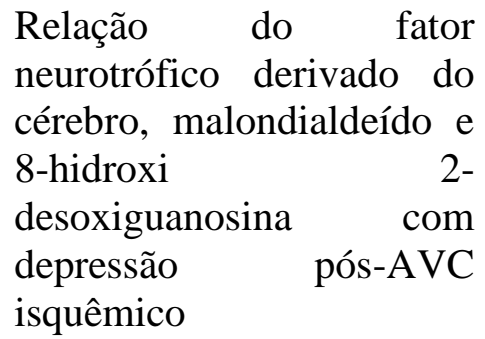 & $\begin{array}{l}\text { Estudo } \\
\text { observacional } \\
\text { conduzido de } \\
72 \text { pacientes } \\
\text { pós-AVC } \\
\text { isquêmico }\end{array}$ & $\begin{array}{l}\text { Os níveis de BDNF no AVC agudo } \\
\text { foram significativamente mais } \\
\text { baixos no grupo de depressão do } \\
\text { que no grupo sem depressão }(p \\
<0,05)\end{array}$ \\
\hline
\end{tabular}

Fonte: dados da pesquisa, 2021.

Os autores (12) relata a relevância dos enfermeiros nos pacientes que apresentam sequelas do AVE, dado que estão relacionados com o planejamento da alta hospitalar e com o seguimento desses pacientes, visto que há a necessidade da continuidade dos cuidados no domicílio, adaptando-o à situação de dependência, além da orientação acerca dos cuidados à saúde e da reabilitação.

\section{DISCUSSÃO}

A equipe de enfermagem possui papel primordial na assistência ao cliente acometido por AVE, sendo assim, quando há uma melhor compreensão da condição clínica indivíduo, bem como das possíveis complicações geradas pelo acidente, consequentemente as condutas assistenciais voltadas para esses pacientes serão satisfatórias e mais assertivas ${ }^{(5)}$.

Dessa forma, o enfermeiro é o responsável por realizar a triagem de todo paciente com sinais e sintomas de AVE. Nesse sentido, como medida inicial para conter o agravamento do evento, inicia-se a terapia trombolítica, especificamente o trombolítico co-ativador de plasminogênio tecidual recombianante (r-TPA), que necessita ser administrado até 60 minutos da admissão do cliente na instituição hospitalar. Além disso, cabe a equipe multiprofissional realizar monitorização contínua e prevenção de complicações ${ }^{(14)}$.

Principal causa de incapacidade a longo prazo, o AVE, origina diversos graus de incapacidade crônica ${ }^{(12)}$. Assim, os principais 
sinais e sintomas apresentados pelo indivíduo acometido pelo AVE envolvem desde o início súbito focal com perdas de força a alterações do nível de consciência. Diante disso, muitos clientes apresentam dificuldade de executar Atividades Básicas de Vida Diária (ABVD) e/ou Atividades Instrumentais de Vida Diária (AIVD), como falar ou compreender, paralisia em um lado específico do corpo, perda visual e cefaleia persistente ${ }^{(7,12)}$.

Como forma de diagnóstico de AVE, utiliza-se a escala de Cincinnati para encontrar a confirmação de ao menos uma das três principais alterações percebidas no corpo, sendo elas: queda facial, fala anormal e debilidade dos braços ${ }^{(8)}$. Desse modo, podese citar, as características percebidas por meio da escala, sendo assim, descritas abaixo ${ }^{(15)}$ :

Queda facial: pede-se para o usuário mostrar os dentes ou sorrir. Quando está sem alteração, ambos lados da face se movem igualmente, mas caso um lado da face não se mova tão bem quanto o outro, é indicativo de anormalidade.

Debilidade dos braços: solicita-se para o usuário fechar os olhos e manter os braços estendidos. Quando normal, ambos os braços se movem igualmente ou não se movem. Mas, se um braço não se move ou cai baixo, quando comparado com o outro, esta anormal.

Fala anormal: solicita-se que o usuário diga, por exemplo, "o rato roeu a roupa do rei de Roma”. Caso esteja normal, ele usará as palavras corretas, com pronúncia clara. Se estiver anormal, a pronuncia palavras é ininteligível, usando palavras incorretas ou sendo incapaz de falar.

Pacientes com alterações físicas decorrentes do AVE necessitam de uma maior assistência para sua reabilitação e reinserção no meio social. Diante disso, esses pacientes e seus familiares devem ter sua assistência planejada pelos profissionais de saúde, ofertando auxílio, escuta ativa, diálogo e suporte emocional - onde este auxilia o paciente a vencer o medo do pós-AVE ${ }^{(8)}$. Assim, esta pesquisa destaca a relevância da assistência de enfermagem quanto à necessidade de avaliação do suporte biopsicossocial prestado ao paciente, bem como pelas orientações dadas durante a alta hospitalar (administração de medicamentos; sobre os cuidados com a higiene, a alimentação, as incontinências, dentre outras), observando as peculiaridades do quadro de saúde de cada paciente ${ }^{(16)}$.

\section{CONCLUSÃO}

Sabe-se da importância da assistência de enfermagem para identificação precoce do AVE, visto que geralmente são os primeiros profissionais a terem contato com o paciente através da triagem. Consequentemente, com a rápida detecção é possível que uma conduta seja tomada logo em seguida, juntamente com 
a equipe multidisciplinar, reduzindo a morbimortalidade do enfermo. Dado isso, observa-se a necessidade desses profissionais conhecerem os sinais e os sintomas que indicam a suspeita diagnóstica de AVE, a exemplo disso, a escala de Cincinnati contém uma fácil aplicabilidade e interpretação. Ademais, o enfermeiro além de realizar o cuidado no ambiente hospitalar, também intervém na recuperação do paciente, seja por medidas de autocuidado seja por orientações.

\section{REFERÊNCIAS}

1. Cumbler MDE. In-Hospital Ischemic Stroke. The Neurohospitalist, 2015; 5(3):173-81.

2. Faria ACA, et al. Percurso da pessoa com acidente vascular encefálico: do evento à reabilitação. Rev. Bras. Enferm., 2017; 70(3): 520-28.

3. Graça CSG. Qualidade na abordagem e tratamento do doente com Acidente Vascular Cerebral Isquémico. Escola Nacional de Saúde Pública, Universidade Nova de Lisboa. Tese de Doutorado; 2019.

4. Silva ES, et al. Prevalência e fatores de risco associados ao acidente vascular cerebral em pessoas co hipertensão arterial: uma análise hierarquizada. Revista de Enfermagem Referência, 2020; 5(3): 1-8.
5. Grilo JNF. Melhoria da eficácia das intervenções do enfermeiro especialista de enfermagem de Ctação na promoção do autocuidado nos doentes com AVC. 2018. Universidade de Évora. Dissertação de Mestrado, 2018.

6. Nunes DLS, Fontes WS, Lima MA. Cuidado de Enfermagem ao Paciente Vítima de Acidente Vascular Encefálico. Revista Brasileira de Ciências da Saúde. 2017;21(1):87-96.

7. Silva DN. Cuidados de enfermagem a vítima de Acidente Vascular Cerebral (AVC). Monografia, Universidade Federal do Tocantins, 2020.

8. Meneses PS, et al. Abordagem fisioterapêutica no tratamento de AVC. Jornada de Fisioterapia do Sertão Central, 2019; 1(1).

9. Lima ACMACC; et al. Diagnósticos de enfermagem em pacientes com acidente vascular cerebral: revisão integrativa. Rev. Bras. Enferm. jul./ago. 2016.

10. Saltman AP, et al. Care and outcomes of patients with in-hospital stroke. JAMA neurology, 2015;72(7): 749-755.

11. Cumbler E, et al. Quality of care and outcomes for in-hospital ischemic stroke: findings from the National Get With The Guidelines-Stroke. Stroke, 2014; 1:23138.

12. Lima RJ, et al. Resiliência, capacidade funcional e apoio social de pessoas com 
sequelas de acidente vascular encefálico. Rev. Eletrônica de Enfermagem, 2020; 22.

13. Syafrita Y, et al. Relationship of brain-derived neurotrophic factor, malondialdehyde, and 8-Hydroxy 2Deoxyguanosine with post-ischemic stroke depression. Dementia \& Neuropsychologia, 2020; 14(1):41-6.

14. Souto RSF, et al. Intervenção de enfermagem no paciente com acidente vascular encefálico isquêmico no setor de emergência. Rev. de Iniciação Científica e Extensão, 2019; 2(4):235-240.

15. Alves MG, et al. Conhecimento dos enfermeiros da atenção primária à saúde sobre a escala de Cincinnati. Rev. Atenas Higeia, 2019; 1(1):35-40.

16. Lima RJ, et al. Capacidade funcional e suporte social para pessoas afetadas por acidente vascular cerebral. Rev. Bras. Enferm. 2019;72(4):jul./ag.

Submissão: 2021-01-16

Aprovado: 2021-05-30 